\title{
PERSPECTIVAS EN LA LUCHA CONTRA LA OBESIDAD COMO PROBLEMA DE SALUD PÚBLICA
}

\author{
PERSPECTIVES INTHE FIGHT AGAINST OBESITY AS A PUBLIC HEALTH PROBLEM
}

\author{
Miguel Malo1,a
}

La obesidad, cuya prevalencia en el mundo se ha duplicado en los últimos 30 años, es un enorme problema que estará cada vez más presente en la agenda de salud pública. Para el 2014, los datos de OMS indican que 39\% de las personas adultas de 18 o más años tenían sobrepeso, y el $13 \%$ eran obesas ${ }^{(1)}$.

Según la OPS este es un problema particularmente grave en la región de las Américas. Hoy en día "la obesidad y el sobrepeso han alcanzado proporciones epidémicas" más del $60 \%$ de los adultos tienen sobrepeso o son obesos en la región y, lo más preocupante, es su crecimiento en poblaciones menores. Entre el 20 y el $25 \%$ de los niños y adolescente están afectados por el sobrepeso o la obesidad en Las Américas ${ }^{(2)}$.

De allí la pertinencia de que la Revista Peruana de Medicina Experimental y Salud Pública retome el tema de obesidad en su sección de simposio. En el presente número se presentan los aportes de Del Aguila y de Diez-Canseco, Saavedra-Garcia. con un análisis del panorama de la obesidad y sus factores de riesgo en el Perú ${ }^{(3,4)}$, y que muestran un alarmante incremento de sobrepeso y obesidad en la población peruana, con valores que se han duplicado en el grupo de niños de 5 y 9 años entre el 2008 al 2014. Luego, los autores discuten los factores que están asociados a esta epidemia y, citando diversas fuentes, confirman su relación con el cambio de hábitos alimentarios dado principalmente por la incorporación masiva en la dieta de alimentos y bebidas listas para consumir con alto contenido de grasas saturadas, azúcar y sal, en detrimento de productos naturales como frutas y verduras. Esto, en el marco de los llamados ambientes obeso génicos caracterizados por el fácil acceso y disponibilidad de alimentos no saludables, su enorme publicidad, que generalmente contrasta con mayores precios de productos naturales; dando como resultado dietas altas en calorías y de bajo valor nutricional. Todo ello sumado a condiciones de planificación urbana que impiden o dificultan la actividad física favoreciendo el sedentarismo en la población.

Aunque la revisión de estudios en varios países muestra una relación entre pobreza y mayor riesgo de sobrepeso y obesidad, Del Aguila presenta dos estudios en el Perú que, por el contrario, muestran más riesgo de sobrepeso entre niños con hogares de mayor recurso económico. Aspecto sobre el que, como lo señala el mismo autor, habrá que profundizar el análisis en el Perú (3).

De manera que, la respuesta a la magnitud y la complejidad del problema de la obesidad implica una serie de medidas de políticas, legislación y reglamentación sólidas que permitan combatir esos ambientes obeso-génicos. En tal sentido, el Plan de Acción para la Prevención de la Obesidad y el Sobrepeso en Niños y Adolescentes ${ }^{(5)}$, aprobado por unanimidad en el 53 Consejo Directivo de OPS es un marco de referencia fundamental para la implementación de estas políticas en nuestra región.

Teniendo como referencia ese marco general, en el simposio se presentan un par de propuestas novedosas como insumos para su implementación. Por un lado, Diez-Canseco y Saavedra-Garcia (4), en la segunda parte de su artículo, presenta un abordaje inicial para una propuesta poco explorada. La discusión sobre la calidad nutricional de los programas sociales de complementación alimentaria como los comedores populares, los programas del vaso de leche y Qali Warma, que originalmente han estado orientados a cubrir deficiencias nutricionales. Sin embargo, pueden tener un gran potencial como intervenciones poblacionales para promover una alimentación

\footnotetext{
1 Organización Panamericana de la Salud. Lima, Perú

a Doctor en Medicina; Editor invitado

Recibido: 17/02/2017 Aprobado: 22/02/2017 En línea: 23/03/2017
}

Citar como: Malo M. Perspectivas en la lucha contra la obesidad como problema de salud pública [Editorial]. Rev Peru Med Exp Salud Publica. 2017;34(1):5-6. doi: 10.17843/rpmesp.2017.341.2760 
saludable. Cabe destacar particularmente, los resultados preliminares del proyecto "Cocinando para la salud", que constata el interés de las usuarias de los comedores populares para incorporar frutas y verduras en su oferta de alimentación.

Por otro lado, y reconociendo que experiencias de política pública en la modificación del etiquetado nutricional son relativamente nuevas en la región, hemos incluido el artículo de Freire et al ${ }^{(6)}$, sobre un primer esfuerzo de evaluación de la política sobre el etiquetando nutricional de alimentos procesados en el Ecuador, implementada a partir del 2014. Este consiste en una investigación cualitativa sobre los conocimientos, actitudes y prácticas relacionadas con el etiquetado nutricional de los envases de alimentos procesados, denominado semáforo nutricional, por su referencia a las advertencias sobre el contenido de grasas, azúcar y sal con los colores del semáforo: rojo, amarillo y verde. Sus conclusiones indican que el semáforo nutricional ha sido positivo para mejorar en la población el conocimiento y comprensión del contenido de grasas, azúcar y sal en los alimentos procesados. No obstante, insiste en la necesidad de entender esta medida como parte de una política pública más amplia de regulación y control sobre los alimentos procesados y de promoción de alimentación saludable.

Finalmente, y considerando que la respuesta a la obesidad y sus consecuencias en ultimo termino acaban en los servicios de salud y que, por tanto, los servicios van a tener cada vez mayor presión financiera para responder a esta demanda, se hace cada vez más necesario poder estimar apropiadamente los costos de dichos servicios. No solamente para saber cómo financiarlos, sino para poder contar con información financiera que nos permita abogar por los aspectos preventivos y promocionales como los más importantes y efectivos para enfrentar esta epidemia.

Por ello, se incluye en el simposio un último artículo de Castillo et al. ${ }^{(7)}$, quien propone una metodología para estimación de costos de los servicios de atención a obesidad, hipertensión y diabetes. Esta metodología pone al alcance de los trabajadores de la salud estos aspectos complejos de economía y financiamiento de la salud, permitiendo que sean capaces de estimar estos costos y de esa manera tengan mejores condiciones de responder a estos nuevos desafíos en los servicios de salud, y tengan instrumentos para generar información útil para abogar por los aspectos preventivos y de protección de la salud.

Esperamos que este simposio que ha pretendido recoger aspectos innovadores, aporte al debate en el Perú sobre la necesidad de ampliar y profundizar las políticas para frenar un problema de salud pública de tal gravedad como la obesidad. De hecho, la comunidad de salud pública debe mantenerse alerta. Tal como lo dijera la Dra. Chang, directora general de la OMS: "La globalización de estilos de vida no saludables no es de ninguna manera un simple problema técnico de la salud pública. Es un problema político. Es un problema de comercio. $\mathrm{Y}$ es un problema de relaciones internacionales. .... Los esfuerzos para prevenir las enfermedades no transmisibles van en contra de los intereses comerciales de operadores económicos poderosos" (8).

El debate, hasta el momento no saldado en el país alrededor de la ley 30021 (ley de promoción de la alimentación saludable) de la que todavía no se tiene el reglamento es un ejemplo de ese conflicto de intereses. De allí, el valor de la revista para colocar estos temas en debate como un espacio privilegiado para abogar desde la salud pública por la salud de la población.

\section{REFERENCIAS BIBLIOGRÁFICAS}

1. Organización Mundial de la Salud [sede Web]. Ginebra: OMS; 2017. [actualizada Junio de 2016; acceso 12 de diciembre de2016]. Obesidad y sobrepeso: nota descriptiva. Disponible en: http://www. who.int/mediacentre/factsheets/fs311/es

2. Organización Panamericana de la Salud [sede Web]. Washington: OPS, 2017. [actualizada 3 de octubre del 2014; acceso diciembre de2016]. Plan de Acción parala Prevención de la Obesidad y el Sobrepeso en Niños y Adolescentes. Disponible en: http://www.paho.org/hq/index.php?option $=$ com_docman $\&$ task $=$ doc_downlo ad\&gid $=28899 \&$ Itemid $=270$ \&lang $=$ en

3. Del Aguila Villar CM. Obesidad en el niño: factores de riesgo y estrategias para su prevención en Perú. Rev Peru Med Exp Salud Publica. 2017;34(1):113-8. doi: 10.17843/rpmesp.2017.341.2773.

4. Diez-Canseco F, Saavedra-Garcia L. Programas sociales y reducción de la obesidad en el Perú: reflexiones desde la investigación. Rev Peru Med Exp Salud Publica. 2017;34(1):105-12. doi: 10.17843/rpmesp.2017.341.2772

5. Organización Panamericana de la Salud [sede Web]. Washington: OPS, 2017. [actualizada 3 de octubre del 2014; acceso diciembre de2016]. Plan de Acción para la Prevención de la Obesidad y el Sobrepeso en Niños y Adolescentes. Disponible en: http://www.paho.org/hq/index.php?option $=$ com_docman\&task $=$ doc_downloa $\mathrm{d} \&$ gid $=28899$ \&Itemid $=270$ \&lang=en

6. Freire WB, Waters WF, Rivas-Mariño G. Semáforo nutricional de alimentos procesados: estudio cualitativo sobre conocimientos, comprensión, actitudes y prácticas en el Ecuador. Rev Peru Med Exp Salud Publica. 2017;34(1):11-8. doi: 10.17843/rpmesp.2017.341.2762.
7. Castillo N, Malo M, Villacres N, Chauca J, Cornetero V, Roedel de Flores K, Tapia R, Ríos R. Metodología para la estimación de costos directosde la atención integral para enfermedades no trasmisibles. Rev Peru Med Exp Salud Publica. 2017;34(1):119-25.doi: 10.17843/rpmesp.2017.341.2774

8. Promoción y Educación para la Salud. Centro de Recursos de Promoción y Educación para la Salud, adscrito al Servicio de Promoción y Educación para la Salud de la Dirección General de Salud Pública de la Consejería de Sanidad y Política Social. [Internet]. Murcia: Dirección General de Salud Pública de la Consejería de Sanidad y Política Social,2013 [consulta diciembre 2016]. Disponible en: http://blogs.murciasalud. es/edusalud/files/2013/07/Discursoapertura-8\%C2\%AA-ConferenciaMundial-de-Promoci\%C3\%B3n-de-laSalud-Helsinki-2013.pdf 